\title{
ENTRE NATURALEZA Y TÉCNICA: UNA CUESTIÓN DE TACTO
}

\author{
Valentina Bulo Vargas \\ Universidad de Santiago de Chile \\ valenbulo@hotmail.com
}

\begin{abstract}
Resumen
Hay dos frases que quisiera comentar aquí: "No hay naturaleza", de Jean-Luc Nancy, y "el ser es el Capital", de Frédéric Neyrat. La intersección de ellas pasa por Heidegger y su idea de Gestell. No es objetivo de este texto hablar "sobre la naturaleza", como lo hicieron los primeros griegos, ni hacer una historia del concepto; más bien intentaremos realizar un trazo entre naturaleza y técnica utilizando los conceptos de Gestell, Ecotecnia y consumación, de Heidegger, Nancy y Neyrat, respectivamente. Este "entre" (al estilo heideggeriano) indicará una figura epocal: uno de los trazos de nuestro mundo globalizado marca esta fusión de naturaleza y artificio.
\end{abstract}

Palabras Clave: Heidegger, Nancy, técnica, naturaleza, ecotecnia.

\section{Abstract}

There are two phrases that I would like to comment: "There is no nature", from Jean-Luc Nancy, and "the being is the Capital", from Frédéric Neyrat. The intersection between both of these concepts passes through Heidegger and his idea of Gestell. It is not an objective of this text to talk "about nature", as the first Greeks did, neither to carry out a history of the concept; rather, what we will try to do is to draw a line between nature and technique using the concepts of Gestell, Ecotecnia and consummation, from Heidegger, Nancy and Neyrat, respectively. This "between" (in Heideggerian style) will indicate an epochal figure: one of the lines of our globalized world marks this fusion of nature and artifice.

KEY WORDS: Heidegger, Nancy, technique, Nature, Ecotecnia.

$\widehat{R A}$

Hay dos frases que quisiera comentar aquí: "No hay naturaleza", de Jean-Luc Nancy, y "el ser es el Capital", de Frédéric Neyrat. La intersección de ellas pasa por Heidegger y su idea de Gestell, curiosamente traducida por Michel Haar en el Canto a la tierra como consumación. El problema real que gatilla esta incursión se vincula con ciertos cuerpos concretos llamados "naturales" y una gran ebullición discursiva sobre la "naturaleza" y su defensa, en el marco de la violenta explotación y aniquilación de nuestros "recursos naturales".

La naturaleza, como concepto, sigue hoy operando como fundamento en gran parte de los distintos discursos "de actualidad"; el uso de este concepto dentro de estos discursos determina a la naturaleza principalmente como un tipo de recurso, son los 
recursos naturales, o sea, algo que "se define" por su disponibilidad, para ser extraído, explotado y consumido -el otro tipo de recurso, el recurso humano, tiene similares características, volcadas a la extracción, explotación y consumo de su fuerza. También operan de este modo aquellos discursos que pretenden velar por el cuidado de la naturaleza, como un lugar que se consume turísticamente, o se nos dan a consumir los "desastres naturales" a través de la televisión y la web: constatamos allí "la fuerza de la naturaleza".

Uno de los orgullos de "la humanidad" que actúa como símbolo del desarrollo ha sido el incluir la naturaleza dentro de los derechos humanos. Expresiones como "reservas naturales" o "patrimonio natural", como parte del patrimonio de la humanidad, apuntan en esa dirección: tomar un fragmento de tierra y restringir su uso al de la conservación y protección en vistas del futuro de la humanidad.

No es objetivo de este escrito hablar "sobre la naturaleza", como lo hicieron los primeros griegos, ni hacer una historia del concepto, desde la physis a la naturaleza moderna, más bien intentaremos realizar un trazo entre naturaleza y técnica utilizando los conceptos de Gestell, Ecotecnia y consumación, de Heidegger, Nancy y Neyrat, respectivamente. El ámbito de este escrito está entre naturaleza y técnica, el "entre" (al estilo heideggeriano) indica una figura epocal: uno de los trazos de nuestro mundo globalizado marca esta fusión de naturaleza y artificio.

$\mathrm{Si}$, como afirma Nancy, "no hay naturaleza" en términos de un orden previo al construido por el hombre, al orden cultural tecnificado, tampoco hay "técnica pura", como algo escindido, separado y superpuesto a la naturaleza. "Si la técnica es el desplazamiento de la naturaleza, nosotros no podemos ver su contrario, o bien, tendríamos que saber pensar un retorno de la naturaleza, por ella misma, en su contrario: ¿pero no sería esto volver a una dialéctica allí donde nosotros esperamos, inevitablemente una "segunda naturaleza"? Es necesario entonces pensar de otro modo. Si la técnica da el sentido de la naturaleza a partir de la cual ella se construye y que ella destruye al mismo tiempo, esto quiere decir que no es más de hecho posible hablar de naturaleza, ni por consecuencia tampoco, en estos términos, de 'técnica'”(Nancy 2010).

Ya desde los años 30, Heidegger hablaba de la era de la técnica, aunque no fue hasta los años 50 que pasó a ser uno de los ejes de su pensamiento tardío; entre los muchísimos discursos sobre la técnica, el discurso heideggeriano destaca por su análisis ontohistórico (Rodríguez 2006, p. 160), que es el que trabajaremos aquí, aunque con una "pequeña vuelta de tuerca", que consiste en el uso de la diferencia ontológica como diferencia colonial. Realizar un análisis ontohistórico de la técnica quiere decir, en primer término, que la técnica no se entiende como la suma de artefactos técnicos que de modo omnipresente funcionan en las urbes "desarrolladas" de hoy, frente a las cosas naturales, como los árboles, el agua o incluso la fuerza del agua de un tsunami (¿cómo clasificar entonces al gato de mi casa o a la energía atómica de una central?), el análisis ontohistórico de Heidegger apunta a ciertas relaciones que él llama fundamentales (yo diría dominantes), que dan "una figura técnica" a los incluidos en dicha relación. Una plantación de bosques es técnica no porque hayan sido plantados por el hombre, sino porque la configuración de relaciones entre el sol, el agua, los árboles y el hombre 
contiene los trazos propios de la figura de la técnica, que consiste en una cadena de producción-explotación, almacenamiento, distribución y consumo de energía de la naturaleza, esta cadena para Heidegger es un emplazamiento o provocación, "una provocación que pone ante la naturaleza la exigencia de suministrar energía que como tal pueda ser extraída y almacenada" (Heidegger 1994, p.18). En este sentido, la técnica moderna puede ser entendida como una cierta violencia, una relación de dominio con eso que Heidegger llama naturaleza.

En segundo lugar, y se articula con lo anterior, el que el análisis de la técnica sea ontohistórico significa que atañe directa y primariamente a la verdad. La técnica es un modo de la verdad, por eso Heidegger dedica tantas páginas y se da tantas vueltas en el esfuerzo (y forcejeo) de mostrar la técnica como un modo de "desocultación provocante", porque la técnica es una cristalización de sentido, del sentido del ser. Esto quiere decir, en términos concretos, que comprendemos el mundo técnicamente, que la verdad tecnocientífica nos aparece como "naturalmente verdadera", pues la técnica se corresponde con la actual figura de despliegue del ser, es lo que Heidegger denomina Gestell.

\section{Atendamos a este texto:}

"Lo que despliega a los montes originariamente en surcos montañosos atravesándolos en su plegada unión es aquella reunión que nosotros llamamos cordillera.

Nosotros llamamos ánimo a aquello que reúne originariamente, desde donde se despliegan las tonalidades según las cuales nosotros sentimos tal o cual humor.

Nosotros llamamos hoy Ge-stell a ese provocante reclamo que reúne al hombre para establecer la sobreexposición [de las cosas] en forma de stock" (Heidegger 1994, p. 23).

El texto anterior puede ser trabajado como una fórmula: son tres momentos en que hay algo que se define (se despliega) como un principio ordenador (reunión originaria) que le otorga un determinado carácter a un ámbito de entes, los determina como tales, les da un sentido, una figura: la cordillera es el principio ordenador que despliega a las montañas según sus surcos, el ánimo es el principio ordenador que despliega a nuestros humores según sus tonos y Gestell es el principio ordenador que despliega la sobreexposición de las cosas como stock según el provocante reclamo.

Gestell entonces es un modo de reunir, de apertura del sentido, que consiste en una necesidad (no una necesidad lógica, ni un apremio), una exigencia humana que se relaciona con las cosas y los otros hombres como existencias expuestas y dispuestas. Eso es lo que nos provoca, el que las cosas se nos presentan como disponibles. Sin pretender hacer una traducción, llamaré disponibilidad a Gestell, justamente porque alude a ese modo originario de abrírsenos el mundo como disponible, lo que nos hace hablar de los hombres como recursos humanos (también como capital humano) y de la naturaleza como recursos naturales. Desde esta perspectiva, lo que primero somos es un recurso. Esto determina el carácter de presentársenos las cosas, que como presencia es una sobreexposición, en forma de stock. Las cosas están allí, listas para ser requeridas. En un documental llamado El Espiral, Armand Mattelart analoga las 
"riquezas naturales" de Chile (salitre, cobre, agua) a una cortesana, que pasa de los brazos de unos extranjeros a los brazos de otro, tierra dispuesta y abierta para los brazos del hombre, tierra sobreexpuesta y sobreexplotada.

Lo importante para Heidegger es el carácter ontológico de este trazo; Gestell es una figura del Ser, por eso es un modo de reunir y es apertura del sentido, como figura del ser corresponde al ser mismo en cuanto retirado, sustraído de la historia. Dicho de otro modo, las cosas nos aparecen como fundamentalmente disponibles para el hombre porque aparecen históricamente vacías de ser, aparece solo el ente vaciado de otro sentido que no sea su disponibilidad. La diferencia ontológica es aquí también lo que está en juego. Gestell es el modo de relación entre el ser y el ente bajo la figura del retiro del Ser, "sólo hay entes, en la forma de puros objetos requeribles por la técnica, y el ser, si es que aún tiene algún sentido esa palabra, no es otra cosa que la forma como aparecen los entes, su pura disponibilidad, su puro estar dispuestos para su uso (como puede observarse, la visión cotidiana del mundo descrita en Ser y Tiempo responde perfectamente a la situación de la existencia en la época técnica)"(Rodríguez 2006, p. 265). Como figura del ser la disponibilidad hace aparecer a los entes como mercancías y al hombre como animal de trabajo.

La diferencia ontológica toma la figura del olvido de la diferencia y es en este aspecto que se puede comprender la distinción de J. Acevedo sobre las dos naturalezas en Heidegger, la naturaleza calculable y la naturaleza natural(Acevedo 1999, pp.106110); mientras la naturaleza calculable se corresponde con la disponibilidad, con la figura actual del ser como retiro, la naturaleza natural se corresponde con un ámbito de la figura propia del ser como Ereignis, eso que en otros textos llama la tierra. La naturaleza natural sería la figura propia del Ereignis, en la perspectiva de la tierra, lugar para el habitar de los mortales, a los que corresponde salvar la tierra. Las dos naturalezas se corresponden con las dos figuras del ser como Ereignis y Gestell. La naturaleza calculable es la naturaleza producida por la figura técnica y conceptuada teóricamente por las ciencias exactas o ciencias "de" la naturaleza, es decir, es la naturaleza como producción teórica de la Gestell, las ciencias en este sentido serán un acoplado al engranaje técnico, se trata más bien de un "aparato tecnocientífico" con apariencia de ciencia natural aplicada. Desde aquí Heidegger definirá la naturaleza como una trama efectiva y computable de fuerzas, nuestro almacén principal de stock de energía (Heidegger 1994, pp. 25 y 30).

Claramente, Heidegger pone el acento en el aparecer de las cosas como disponibles, y ese análisis lo inserta en el ciclo de producción-explotación, almacenamiento, distribución y consumo, pero no trabaja el ciclo como tal. Frédéric Neyrat reclama la necesidad de leer este ciclo desde su fin, o sea, desde el consumo (Neyrat 2008, p. 20), Neyrat toma la traducción de Haar de Gestell por consumación para hacer emerger desde ella el triple sentido de consumir, aniquilar y consumar. Si Gestell es una cara del ser en el sentido de hacer aparecer los entes como disponibles, es porque Gestell es lo que fuerza a ser de los entes entes para el consumo, de ahí su disponibilidad. Salmones criados en cultivos, semillas diseñadas genéticamente para su consumo (sin reproducción) son ejemplos evidentes, sin decir nada de los aparatos "propiamente" tecnológicos, que tienen trazado su ciclo vital para una pronta consumación y proliferación en la diversificación 
del consumo. Lo grave es la lógica de funcionamiento que se dirige, como un hoyo negro, hacia su consumación; se trata en este sentido de un ciclo que se cierra sobre sí mismo, el consumo es su consumación, la consumación del ser. Neyrat identifica la cadena que Heidegger trabaja en Gestell con la "lógica del capital", pues habría una correspondencia entre el ser como Gestell y el capital. Este hacer coincidir ser/capital está muy lejos de la mera pretensión de hacer una interpretación de Heidegger, lo que hace Neyrat es algo más alquímico, junta estos conceptos-fuerza y los hace estallar en su pensamiento explosivo.

Hacer corresponder el ser y el capital, hacerlos unívocos tiene la ventaja de ampliar y repensar el capital desde la ontología, no ya solo desde la sociología, economía, etc.; es pensar el capital como una cierta lógica, un modo de reunir el sentido desde donde puede aparecer eso que llamamos nuestro mundo; producción, distribución y consumo como nuestro "ciclo natural" de organización de sentido es justamente ontopolítica, porque politiza al ser y ontologiza la política, piensa la polis desde lo ontológico, como un problema que involucra a "todos los entes" y no ya solo a los ciudadanos, donde el hombre deja de ser el centro del problema para dejar lugar a la tierra y a los otros cuerpos.

La lógica del capital es aquella política que se identifica completamente con la economía, "la economía es la invención propia del capitalismo, esa es la singularidad que lo define... la economía es una política en la medida en que su objeto es borrar la posibilidad misma de la política...en la medida que es capaz de monopolizar sin resto la forma de la elección" (Aspe 2011, p. 30). Si le otorgamos rango ontológico a una afirmación como esta damos con un matiz especial a lo que Heidegger trabajó con la figura de los entes como mercancías o stock, pues el ser absorbible en procesos económicos confiere ahora un rango existencial: existimos en la medida en que participamos de la racionalidad económica. Esta racionalidad económica como condición de posibilidad de la existencia atañe a todo lo existente.

Atañe a los hombres como existentes en la medida en que están disponibles; es lo que ya Heidegger señalaba al hablar de los hombres como animales de trabajo, como recursos humanos, como "material humano avaluado según el puesto que ocupe en el dispositivo de la producción y según su eficacia y rendimiento en él" (Acevedo 1999, p. 110), es lo que hoy definimos como "capital humano". También atañe a los hombres como consumidores, es lo que Neyrat llama "imperativo de consumo", que nos dice "consume, consume, todo lo que tú quieres, tu vida y tu ser, tus relaciones si quieres, elige tu mundo de consumición, pero no toques la propiedad privada" (Neyrat 2004, p. 134); este imperativo asegura su cumplimiento al hacer del hombre un ser endeudado, donde la deuda tiene el rango de deuda existencial, que nos arroja al ciclo del consumo.

Si se establece la lógica del capital como condición de posibilidad de la existencia (de todos los entes, no solo los hombres) en el sentido descrito, queda literalmente fuera de la existencia, fuera del mundo todo lo que esta racionalidad no absorbe o desecha. Al ciclo natural de producción agregamos el desecho entendido como flujo residual. Los hombres-desecho de la racionalidad económica literalmente quedan fuera de la existencia; en Latinoamérica se ha dicho alguna vez que el único modo de existir para todos aquellos que quedan fuera del mundo globalizado es en el momento en que ellos 
dan muerte o son masacrados. No es casual el derramamiento de sangre que se produce en la zona de desgarro entre el mundo y el no mundo, llámese frontera de México, los bordes de la selva amazónica o la no-tierra mapuche. Respecto a la tierra, ésta queda convertida en "el desecho del proceso de simbolización del globo", mundialización "que se edifica sobre la aniquilación de la tierra" (Neyrat 2004, p. 182).

Desde esta perspectiva, la diferencia ontológica heideggeriana toma ahora el matiz de la diferencia mundial, que consistiría en la relación diferencial "entre el mundo en tanto que producido (globalizado, informatizado) y el mundo en tanto que se sustrae a la producción. La diferencia mundial es la diferencia óntico-ontológica pensada a partir del mundo... mundo como realidad asubjetiva y desencarnada; el mundo no es un sujeto, no es ni Dios, ni el hombre, ni la madre naturaleza" (Neyrat 2004, p. 17).

Si bien la identificación del ser y el capital abre una perspectiva ontopolítica necesaria a la hora de pensar la cuestión de la técnica y la naturaleza, considero que adolece de la misma totalización heideggeriana, solo que la demoniza, y es que bajo el imperio del capital justamente quedan subsumidas todas las diferencias, hasta no existir. Neyrat alimenta en este punto, me parece, un esquema interpretativo-paranoico que termina reafirmando vía negativa la hegemonía del ser a través de la diferencia ontológica. Frente a un esquema así restaría una salida total, como el nuevo inicio histórico heideggeriano a partir de la experiencia fundamental de la reserva y el desasimiento, que equivale a la fundación de la naturaleza técnica y manipulable a partir de la naturaleza natural. En este juego entran aquí también todas aquellas propuestas ecológicas (como la ecología profunda) que apelan a la naturaleza como bien.

Sin negar lo anterior, más bien retomando la importancia de realizar el análisis de la técnica desde una perspectiva ontopolítica y, por tanto, a la "luz" del capital, es necesario pensar con tacto: ontologizar la técnica, hacer de la técnica el acontecimiento epocal, como hace Heidegger, nos permite trabajar la realidad con caracteres desnaturalizados, es decir, con principios y fines que no nos son dados ni en forma mítica ni positiva (natural), desde allí es que toda cuestión técnica es política, porque porta en sí misma la decisión y de allí que toda técnica es ontológica, porque es un carácter fundamental de nuestro mundo. De hecho, y es la propuesta que examinaremos ahora, la técnica es "el mundo haciéndose mundo" (Nancy 1993, p. 66), sin un sentido fuera de sí, simplemente "nuestro mundo", ya desnaturalizado. Por esto, el mundo es nuestra creación, el peso lo llevamos nosotros, los cuerpos del mundo.

Nancy llamará ecotecnia al trazo (menos que figura) de nuestro mundo, la ecotecnia es un trazo ambiguo, mejor dicho, un trazo múltiple. Expongo algunas de las definiciones de ecotecnia (las dos primeras son en rigor definiciones de la técnica, entendiendo aquí que la ecotecnia es el nombre ontológico de la técnica):

1. "La técnica es Ereignis, el evento apropiante de la existencia finita en cuanto tal. Ella expone de un mismo golpe la cuestión de una existencia finita como tal y de su apropiación también finita" (Nancy 2002, p. 76).

2. "La técnica es el acontecimiento desnaturalizante" (Nancy 2002, p. 127). 
3. "[La ecotecnia es] el mundo como creación: una techne sin principio ni fin, ni otros materiales fuera de ella misma...como lo que espacía y que difiere la physis hasta los confines del mundo" (Nancy 1993, p. 66).

4. Exposición del mundo en la creación de los cuerpos, creación como techne de los cuerpos (Cf. Nancy 2006, p. 79).

5. Efectividad del (des)orden mundial con la doble cara de 'técnica planetaria' y 'economía-mundo' que hace del mundo hoy un mundo sin razón, sin fin y sin figura (Cf. Nancy 1996, p. 158).

Las dos primeras definiciones corresponden a definiciones ontológicas de la técnica muy cercanas a las expresiones heideggerianas, pues ellas apuntan al Ereignis y lo corrigen, lo toman y lo difieren hacia el acontecimiento desnaturalizante. La primera definición está en el texto llamado "lo insacrificable", con lo que ya podemos adelantar que lo que le interesa corregir a Nancy es cualquier rastro de sacrificio en los conceptos heideggerianos, "es exactamente en ese punto que hay que corregir, sin descanso, a Bataille y a Heidegger. Corregirlos, es decir, retirarles la menor inclinación hacia el sacrificio. Porque la inclinación hacia el sacrificio, o por el sacrificio, está siempre ligada a la fascinación de un éxtasis tornado hacia Otro o hacia un Afuera absolutos" (Nancy 2002, p. 79). Nancy toma y acepta la identificación heideggeriana de Gestell y Ereignis, pero no con Otro Ereignis fuera, no con Otra naturaleza más natural, a eso llama existencia finita en su apropiación finita, lo desnaturalizante apunta justamente a que no hay un afuera absoluto, ni como principio ni como fin ni como origen, por ello no hay posibilidad de sacrificio.

Aquí encontramos la primera gran diferencia con Heidegger: en Heidegger, "la esencia de la técnica" no es técnica porque no es del plano óntico, sino ontológico, a eso apunta con Gestell, pero en Nancy, anulada la diferencia ontológica habría que decir que "la esencia de la técnica" es técnica, pues no indica nada fuera de sí, eso es justamente desnaturalizar. Desnaturalizar es una expresión inexacta, en el sentido de que no hay una naturaleza previa que se desnaturaliza, "la técnica no re-forma una Naturaleza, ni un Ser, en un Gran Artificio: sino que ella es el "artificio" (y el arte) del hecho de que no hay naturaleza" (Nancy 2002, p. 30).

Ecotecnia será la expresión precisa de este "entre" naturaleza y técnica que consiste en el hacerse mundo del mundo, sin Otro afuera, mundo como nuestro artificio, nuestra creación, creación de los cuerpos en el diferir. Ecotecnia es el nombre para la multiplicidad de trazos entre unos cuerpos y otros, vivos y no vivos, humanos y no humanos, "naturales" o "artificiales". Ecotecnia es el nombre del trato de unos cuerpos con otros, simétricamente; por eso es una cuestión de tacto, de ensamblaje, de técnica, de maña, nos inventamos el cuerpo en el hacer mundo y viceversa. De ahí que una ecología necesariamente ha de ser un planteamiento técnico respecto a la técnica, no hay "naturaleza" que cuidar, en términos de un conjunto de principios externos o dados a nuestro mundo; el problema no está, por tanto, en optar por una priorización de "lo natural" v/s lo técnico, el problema está en nuestra coexistencia dominante con los otros cuerpos. 
En ser singular plural, Nancy plantea la tesis del acabamiento del modelo soberano, que sería aquel modelo que tiene a la guerra por técnica de su propia afirmación. Un acabamiento, sin embargo, que no tiene otro modelo, por eso Nancy habla de una soberanía sin soberanía. El acabamiento de la soberanía tiene que ver directamente con la técnica, soberanía es "la potencia de la ejecución o del llevar a cabo (finition) como tal, absolutamente y sin ninguna otra subordinación a cualquier otra cosa (a otro fin)" (Nancy 1996, p. 146), soberanía sin soberanía es la pura potencia ejecutoria sin fundamento. Nuestro actual mundo mundial estaría trazado por esta ambigüedad, "dominación suprema de aquello que no habría tenido ni el fulgor del origen, ni la gloria del cumplimiento de una presencia soberana; ni Dios, ni héroe, ni genio, y por tanto la lógica del sujeto de excepción, sujeto sin ley de su propia ley, y una ejecución, un llevar a término indefinido e interminable de esta lógica. De esta lenta deportación del mundo en la soberanía sin soberanía, en el llevar a cabo sin fin (finition sans fin), la ecotecnia podría ser la última figura sin figura". Esta figura, afirma, llevaría una triple división entre ricos y pobres, integrados y excluidos, el norte y el sur.

Sin naturaleza u otro principio extrínseco o dado que ordene nuestro mundo, queda la configuración ecotécnica trazada por esta "figura dominante sin figura", que coincide a veces con Gestell o con el Capital pero que aterriza en este trato directo de unos cuerpos-artefactos con otros, en el cruce de singularidades. Es lo que Nancy llama desnaturalización, como acontecimiento histórico, el momento preciso en que "la historia natural se quiebra y se desnaturaliza" (Nancy 2002, p. 116), sin poder ser ya autogeneradora o autotélica. Es el agotamiento de la historia natural como construcción (técnica) occidental.

Esta grave afirmación es sostenida de un modo muy coincidente, aunque desde otro ámbito y con otras raíces, por el historiador indio integrante del Grupo de Estudios Subalternos, Dipesh Chakrabarty, quien habla de un "colapso de la distinción humanista secular entre la historia humana y la historia natural". El umbral desde donde se fusionan ambas historias sería el calentamiento global, que da inicio a una edad de la tierra llamada por algunos científicos (como Crutzen y Stoerner) Antropoceno.

El Antropoceno nombra una edad de la tierra, según la cual ella está determinada por la acción humana, su síntoma sería el calentamiento global, pues marcaría el punto en que la "especie" humana se convierte en un "agente geológico que perturba las condiciones geológicas paramétricas necesarias para la existencia" (Chakrabarty 2009) de la vida en la tierra. Los seres humanos hemos cambiado los procesos físicos más elementales de la tierra. Chakrabarty sitúa su inicio en 1750, que coincide con el despliegue de la ilustración, la idea de libertad, el uso de combustibles fósiles a gran escala, y cuando el análisis del aire que se encontró atrapado en los hielos polares mostró el inicio de la creciente concentración global de dióxido de carbono y metano. "En ningún debate sobre la libertad en el período transcurrido desde la Ilustración hubo ninguna conciencia de la agencia geológica que los seres humanos fueron adquiriendo al mismo tiempo y a través de procesos estrechamente vinculados a su adquisición de la libertad. Los filósofos de la libertad estuvieron principalmente, y es comprensible, preocupados por cómo los seres humanos escaparían de la injusticia, la opresión, la desigualdad, o incluso de la uniformidad impuesta sobre ellos por otros seres humanos 
y sus sistemas artificiales. El tiempo geológico y la cronología de la historia humana se mantuvieron sin relación. Esta distancia entre los dos calendarios, como hemos visto, es lo que los científicos del clima reclaman que se ha desplomado" (Chakrabarty 2009).

Una de las consecuencias importantes para nuestro tema es que el Antropoceno como "acontecimiento histórico" no puede explicarse únicamente desde la figura del capital o de la globalización; si bien "la pared entre la historia humana y natural ha sido traspasada. Es posible que no nos pensemos como agentes geológicos, pero parecemos habernos convertido en uno a nivel de la especie. Y sin ese conocimiento que desafía la comprensión histórica no podemos darle sentido a la actual crisis que nos afecta a todos. El cambio climático, refractado a través del capital global, acentuará, sin duda, la lógica de la desigualdad que se ejecuta a través del dominio del capital; algunas personas ganarán, temporalmente, a expensas de los demás. Pero toda la crisis no puede reducirse a una historia del capitalismo" (Chakrabarty 2009). El análisis crítico de la figura del capitalismo, por tanto, no es suficiente, pero ello no significa dejar fuera este análisis, la tarea asignada por Chakrabarty apunta a una reelaboración conjunta de la crítica del capital, como estrato final de la historia humana y la historia de las especies, como estrato final de la historia natural. "La crisis del cambio climático exige que se piense de forma simultánea en ambos registros, que se mezclen las cronologías inmiscibles de capital y de la historia de las especies" (Chakrabarty 2009).

Esto significa que el Antropoceno, dicho en términos más ontológicos, indica algo más que una interacción entre el hombre y naturaleza, eso ha sido siempre; el Antropoceno es el momento en que ambas historias se hacen una y por tanto se "definen" una desde la otra. La tierra se nombra a partir de la acción humana y "especie humana" se redefine como condición natural, pues en el proceso de dominar otras especies adquieren la condición de fuerza geológica. Esto a todas luces va mucho más allá de una consideración esencialista de la especie y va más allá también de la determinación darwiniana de especies desde su comunidad de descendencia. Desde el Antropoceno, "la humanidad no se hace solamente sobre su cuerpo animal, viviente (bios), sino sobre la Tierra (Gea) toda entera, sobre lo orgánico y lo inorgánico” (Neyrat 2009).

Desde esta tesis se pueden seguir varios caminos, uno de ellos, como lo ve claramente Neyrat, es la constatación del peligro de absolutización de lo humano, su hiperpoder, "remplazar el Helioceno por el Antropoceno es poner peligrosamente lo humano en posición de totalidad" (Neyrat 2009). Pero también es posible, a partir de esta misma "posición de lo humano", abordar la juntura de las dos historias reclamada por Chacrabarty en la crítica del capital y la reelaboración de la historia de las especies y hacerlas remitir a una crítica del humanismo como aquel que determina esa posición de lo humano descrita como antropocéntrica y dominante a partir de su "geopoder".

Me parece que la crítica de Nancy al "sobrehumanismo" de Heidegger y el desplazamiento de la cuestión de la ordenación del mundo (como ecotecnia) al del ensamblaje de los cuerpos resitúa la posición de lo humano: la ecotecnia es una figura de dominación, pero también redistribuye el mundo desde una vinculación simétrica entre singularidades. Tanto la ecotecnia como el antropoceno mantienen esa ambigüedad, el antropoceno es la definición de la tierra a partir de la dominación humana, pero 
también es la fusión de la historia natural y humana. Ambos apuntan a un momento de redistribución del orden mundial que deja atrás dualidades como artificio y naturaleza para abrir otro campo de juego, hasta cierto punto impensable. Nancy habla de una singularidad mundial, como una propuesta ecotécnica al modelo soberano de ordenación mundial, en donde "la singularidad valdría a la vez absolutamente y sin dar ejemplo" (Nancy 1996, p. 166). Esta singularidad es la materialidad de los cuerpos, su realidad, la materia como realidad de la diferencia (Cf. Nancy 1993, p. 96). Más que una operación de descentramiento que le quita el lugar central al hombre en el mundo, Nancy apunta a un omnicentrismo, pues le da el centro a todos los demás en una irreductible pluralidad, por eso es una cuestión de tacto.

\section{Referencias bibliográficas}

Acevedo, Jorge (1999), Heidegger y la época técnica. Santiago: Ed. Universitaria.

Aspe, Bernard (2011), Les mots et les actes. Caen: Ed. Nous.

Chakrabarty, Diepesh (2009), "The climate of history: Four theses". http://www. eurozine.com/articles/2009-10-30-chakrabarty-en.html

Heidegger, Martin (1994), Vorträge und Aufsätze. Tubingen: Ed. Neske.

Latour, Bruno (1998), "La tecnología es la sociedad hecha para que dure", en Domènech Miguel y Tirado Francisco Javier, Sociología simétrica. Barcelona: Ed. Gedisa.

Malabou Chaterine (2004), Le change Heidegger. Paris: Ed. Léo Scheer.

Nancy, Jean-Luc, (2006), Corpus. Paris: Ed. Métailié. (2010), De la struction. Inédito. (1996), Être, singulier pluriel. Paris: Ed. Galilée. (2002), La création du monde ou la mondialisation. Paris: Ed. Galilée. (1993), Le Sens du monde. Paris: Ed. Galilée.

(2002) Un pensamiento finito. Barcelona: Ed. Anthropos.

Neyrat, Frédéric (2008), L'Indemne: Heidegger et la destruction du monde. Paris: Ed. Sens\&Tonka.

(2009), “Climate Turn: L'anthropo-scène, Chakrabarty et l'espèce humaine", en http://www.revuedeslivres.onoma6.com/articles.php?idArt=5 39\&PHPSESSID=92da7d2d.53f358fa3004b038603f3eb (consultado el 9 de agosto de 2012).

(2004), Surexposés. Paris: Ed. Lignes \& Manifeste.

(2005), "L'être, l'image et le capital dans Le Change Heidegger de Catherine Malabou". http://www.ctheory.net/articles.aspx?id=475

Rodríguez, Ramón (2006), Heidegger y la crisis de la época moderna. Madrid: Ed. Síntesis. 